\title{
Clinicopathological features of gastric mucosa associated lymphoid tissue (MALT) lymphomas: high grade transformation and comparison with diffuse large B cell lymphomas without MALT lymphoma features
}

Tadashi Yoshino, Kunihiro Omonishi, Keita Kobayashi, Tomohiko Mannami, Hiroyuki Okada, Motowo Mizuno, Ichiro Yamadori, Eisaku Kondo, Tadaatsu Akagi

\begin{abstract}
Aims-To investigate the clinicopathological differences among gastric low grade MALT lymphomas (low MALT), large B cell lymphomas with low grade components (secondary high grade MALT lymphomas, high MALT), and diffuse large B cell lymphomas without low grade features (primary high grade MALT lymphomas, DLL).

Methods-Clinicopathological and morphological characters of 126 gastric lymphoma cases were studied: 82 cases of low MALT lymphoma including 40 that were surgically resected, 17 cases of high MALT lymphoma including 13 surgically resected, and 27 cases of DLL including 12 surgically resected.

Results-Age ranges were as follows: low MALT lymphoma, 34 to 85 years (mean 59.9); high MALT lymphoma, 53 to 88 years (mean 68.5); DLL, 29 to 83 years (mean 62.3). The average age for low and high MALT lymphomas was significantly different $(p<0.05)$, but there were no differences in other comparisons. There was a female predominance of low MALT lymphoma patients (female to male ratio, 47/35), while for high MALT patients the ratio was almost even (8/9), and for DLL patients there was a male predominance (11/16). Examination of surgically resected material showed that MALT lymphomas had a wider distribution in the gastric wall than DLL.

Conclusions-The findings suggest that at least some of the high grade gastric lymphomas, especially in patients younger than the fifth decade, do not originate from high grade transformation of low MALT lymphomas. It seems to take about one decade at least for high grade transformation of low MALT lymphomas. (F Clin Pathol 2000;53:187-190)
\end{abstract}

Keywords: MALT lymphoma; stomach; transformation

Internal Medicine,

Okayama University

Medical School

H Okada

M Mizuno

Correspondence to:

Dr Yoshino

email:

yoshino@med.okayama-u.ac.jp

Accepted for publication 9 August 1999 included in a new proposal of lymphoma classification. ${ }^{2}$ Aetiologically, gastric low grade MALT lymphoma (low MALT lymphoma) is
Since Isaacson and Wright established the concept of the mucosa associated lymphoid tissue (MALT) lymphoma, ${ }^{1}$ it has been widely closely associated with Helicobacter pylori gastritis. ${ }^{3-6}$ Low MALT lymphomas occasionally progress into high grade lymphomas (secondary or simultaneous high grade MALT lymphomas, high MALT lymphomas). ${ }^{78}$ High grade portions are composed of large lymphoma cells with frequent mitotic figures. High grade MALT lymphomas have been shown to be associated with p53 abnormalities ${ }^{9}$ and overexpression of BCL- $6 .{ }^{10}$ In addition to these high grade MALT lymphomas, gastric lymphomas sometimes show the histology of conventional diffuse large B cell lymphoma without MALT lymphoma features (primary high grade MALT lymphomas, DLL). The histogenesis of these DLL tumours is not yet clear. In the present study, we characterise the clinicopathological features of gastric low and high grade MALT lymphomas and DLL, and suggest that at least some DLL do not originate from malignant progression of low grade MALT lymphomas.

\section{Methods}

CASES, DIAGNOSTIC CRITERIA, AND

IMMUNOHISTOLOGY

We examined 126 cases of gastric lymphomas in our surgical pathology files; 65 of these were surgically resected. As it was recently reported that eradication of $H$ pylori is effective treatment for gastric low grade MALT lymphomas, ${ }^{4-6}$ surgery is now rarely used as first line treatment. Thus most of the resected cases examined in our present study were treated over two years ago. Low grade MALT lymphomas were diagnosed on the basis of previously described criteria. ${ }^{11-13}$ We defined high grade MALT lymphomas as lymphomatous foci containing high grade as well as low grade components (fig 1). Histologically, the high grade component is similar to diffuse large B cell lymphomas. Diffuse large B cell lymphomas without low grade MALT lymphoma features are designated DLL. Eighty two cases were diagnosed as low grade MALT lymphoma, including 40 that were surgically resected; 17 cases were diagnosed as high grade MALT lymphomas, including 13 surgically resected; and 27 cases were diagnosed as DLL, including 12 surgically resected. In most cases with surgically resected material, over 20 blocks were made for histological examination.

All cases were examined immunohistologically with anti-CD20, CD3, CD 45, CD43, 

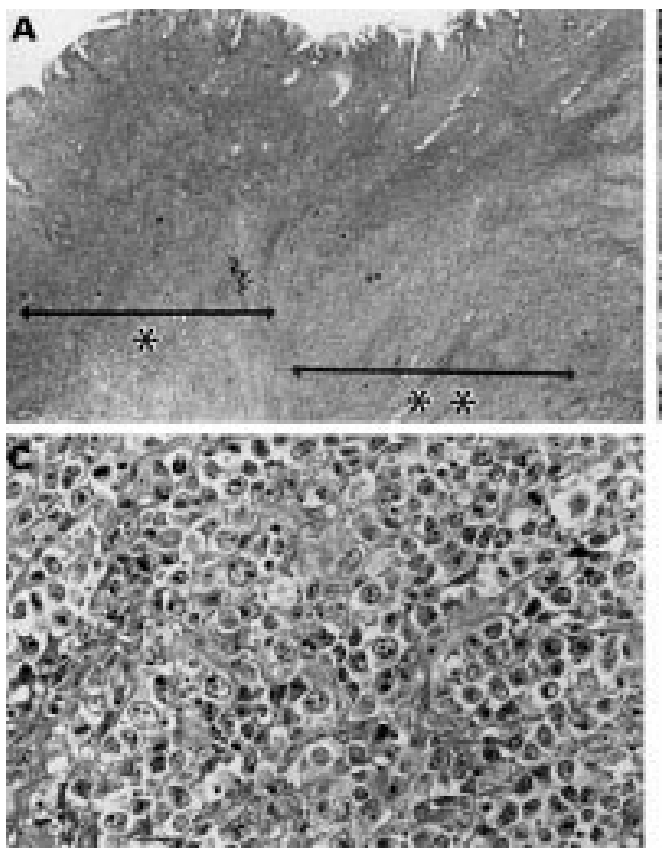

CD45RO, PCNA, cyclin D1, cytokeratin, Igк and $\operatorname{Ig} \lambda$ antibodies for diagnosis and characterisation. Cases in which frozen section were available were also examined with anti-CD5, 10, 19, and 22 antibodies. Immunohistochemical studies were carried out using the avidin-biotin peroxidase complex technique, as previously described. ${ }^{10}$

STATISTICAL ANALYSES

To compare the average ages and sexual differences of examined groups, we used the Student $t$ test and the $\chi^{2}$ test, respectively.

\section{Results}

CLINICAL CHARACTERISTICS

Patients with low MALT lymphoma were aged between 34 and 85 years (mean 59.9); those with high MALT lymphoma were between 53 and 88 (mean 68.5); and those with DLL were between 29 and 83 (mean 62.3) (table 1; fig 2). The average age of the patients with low and high MALT lymphomas was significantly different ( $t$ test, $\mathrm{p}<0.05$ ), but there was no difference in the other comparisons. Interestingly, the age distribution of DLL was much wider than that of MALT lymphomas and was composed of a large group with a normal distribution peaking in the sixties and an additional small group of younger patients aged less than 50 .

As summarised in table 1 , there was a predominance of female patients in the low MALT group (female to male ratio, 47/35), while the sex distribution was almost equal in

Table 1 Summary of the cases examined

\begin{tabular}{lcll}
\hline & $\begin{array}{l}\text { Number of } \\
\text { examined } \\
\text { cases }\end{array}$ & $\begin{array}{l}\text { Sex } \\
\text { (female/male) }\end{array}$ & $\begin{array}{l}\text { Age (years), } \\
\text { range and } \\
\text { (mean) }\end{array}$ \\
\hline $\begin{array}{l}\text { Low grade MALT lymphomas } \\
\text { High grade MALT lymphomas (secondary }\end{array}$ & 82 & $47 / 35$ & 34 to $85(59.9)$ \\
$\begin{array}{l}\text { MALT lymphomas) } \\
\begin{array}{l}\text { Diffuse large B cell lymphomas without MALT } \\
\quad \text { features }\end{array}\end{array}$ & 17 & $8 / 9$ & 53 to $88(68.5)$ \\
\begin{tabular}{l} 
Total \\
\hline
\end{tabular} & 126 & $11 / 16$ & 29 to $83(62.3)$ \\
29 & $66 / 60$ & 29 to $88(61.6)$ \\
\hline
\end{tabular}

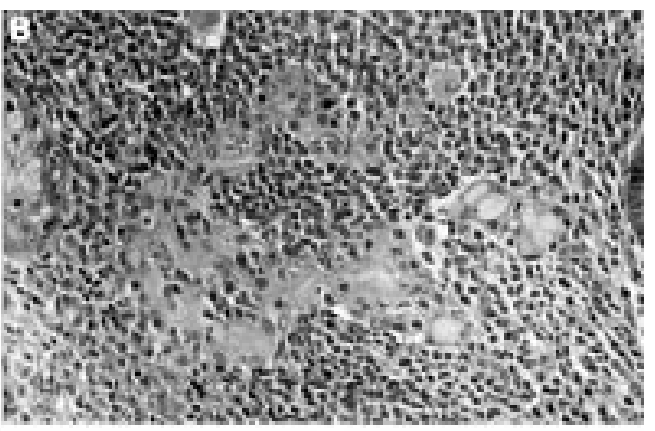

Figure 1 A case of high grade (simultaneous and secondary) gastric MALT lymphoma. ( $A$ ) A low magnification view indicates that the low grade area $\left(^{\star}\right)$ and the high grade area ( $\left.{ }^{\star}\right)$ are directly connected (haematoxylin and eosin, $\times 20$ ). (B) A high magnification view of a low grade MALT lymphoma area showing that the lymphoma cells are medium sized and centrocyte-like, forming a lymphoepithelial lesion $(H \mathcal{E} E, \times 322)$. (C) $A$ high magnification view of a high grade $M A L T$ lymphoma area showing that large sized nucleated lymphoma cells proliferate diffusely $(H \mathcal{E} E, \times 322)$.

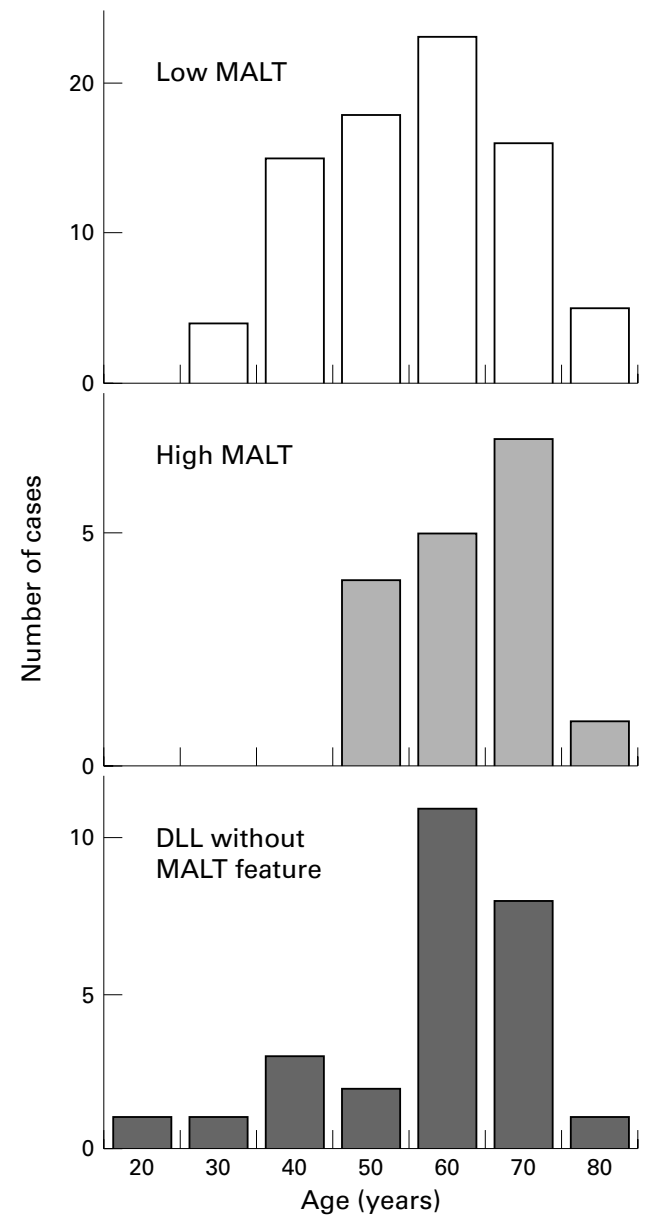

Figure 2 Age distribution of gastric lymphomas. Low MALT, low grade MALT lymphoma; high MALT, high grade $M A L T$ lymphoma; $D L L$, diffuse large $B$ cell lymphoma without MALT lymphoma features.

the high MALT group (8/9). There was a male predominance among the DLL patients (female to male ratio, 11/16); this was particularly marked in the under-fifties, where the 

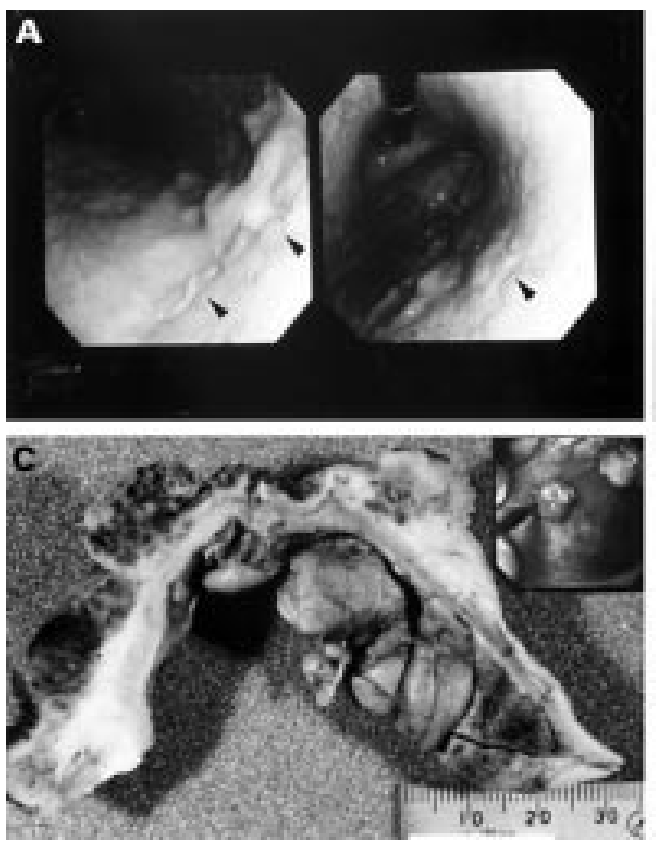

ratio was reduced to $1 / 4$. These difference were not statistically significant, however.

MACROSCOPIC AND HISTOLOGICAL FEATURES Macroscopically, low grade MALT lymphomas showed widespread lesions with multiple irregular shaped erosions or shallow ulcers in 55 cases $(67 \%)$ (fig 3A), large ulcers in 10 cases (12\%) (fig 3B), and swollen mucosal folds with or without multiple polyps in 17 cases $(21 \%)$ (fig 3C). High grade MALT lymphomas often showed large ulcers similar to advanced gastric cancers, or protruded tumours, as well as multiple shallow ulcers. The macroscopic findings of DLL were similar to those of advanced gastric cancer except in two cases which showed multiple ulcers.

Depth, spread, and lymph nodal metastases were examined in surgically resected material. In $77 \%$ of high grade MALT lymphomas and $83 \%$ of DLL, lymphoma cells invaded the muscle layer or more deeply. In contrast, $83 \%$ of low grade MALT lymphomas were confined to the mucosal and submucosal layers (fig 4).

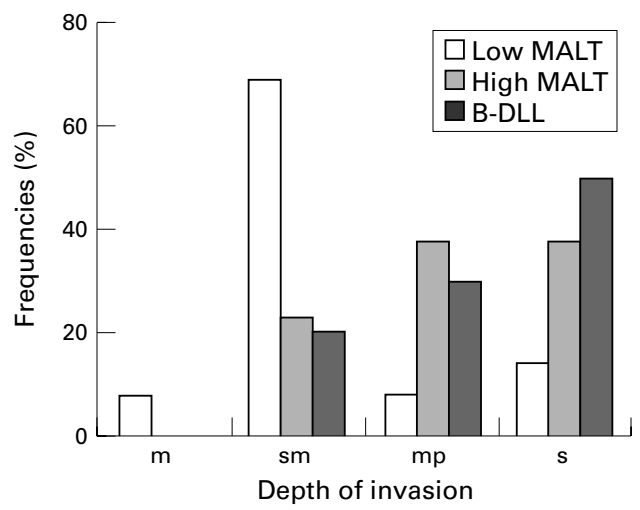

Figure 4 Depth of the gastric walls involved with lymphoma cells; $m$, mucosa; sm, submucosa; $m p$, muscularis propria; s, subserosa and serosa. Low MALT, low grade MALT lymphoma; high MALT, high grade MALT lymphoma; $D L L$, diffuse large $B$ cell lymphoma without $M A L T$ lymphoma features.

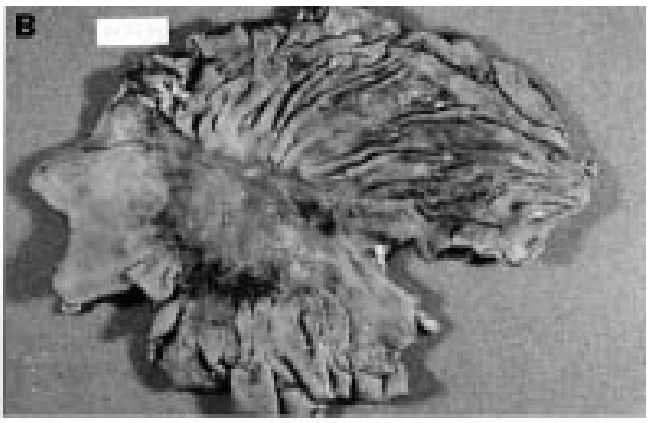

Figure 3 Macroscopic findings of low grade gastric MALT lymphomas. (A) Endoscopic pictures showing irregular shaped shallow ulcers (arrowheads). (B) $A$ resected stomach showing a large scarring ulcer. Lymphoma cells were found on the edge of the ulcer. (C) Cut surface of the stomach with multiple polypoid lesions. The inset panel is the endoscopic view of the same case.

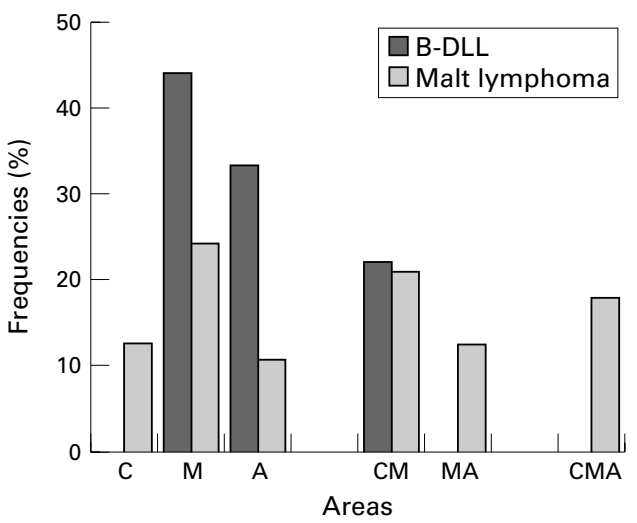

Figure 5 Regional distribution of gastric lymphomas. $C$, upper third; $M$, middle third; $A$, lower third. $C M, M A$, and $C M A$ represent $C+M, M+A$, and the whole area, respectively. $D L L$, diffuse large $B$ cell lymphoma without MALT lymphoma features. MALT lymphomas include both low grade and high grade lymphomas.

The distribution of the lymphomas was investigated by subdividing the stomach into three portions: $C$ (the upper third), $M$ (the middle third), and A (the lower third). MALT lymphomas tended to have multiple lesions and $52 \%$ were distributed in more than one portion. In contrast, $78 \%$ of DLL were confined to a single area (fig 5). The gastric greater curvature was involved in $79 \%$ of all MALT lymphomas, but in only $17 \%$ of DLL. Stump involvement in resected stomachs was detected in five cases of MALT lymphoma (one high grade and four low grade cases), but not in DLL.

The correlation between the depth of the lesions and lymph node dissemination is summarised in table 2. Lymph nodal resection was performed in 60 cases. Lymph node metastases were found in only one of 34 cases $(3 \%)$ that involved only the mucosal or submucosal layers. In contrast, lymph node metastases were often observed in lymphomas invading down to the muscle layer $(45 \%)$ or deeper $(73 \%)$, irrespective of histological type. 
Table 2 Relation between depth of the gastric wall involved with lymphoma and lymph node dissemination

\begin{tabular}{lcc}
\hline Depth of the gastric wall & No of lymphoma cases & $\begin{array}{l}\text { No (ratio) of cases with lymph } \\
\text { node dissemination }\end{array}$ \\
\hline Mucosa & 4 & $0(0 \%)$ \\
Submucosa & 30 & $1(3 \%)$ \\
Muscularis propria & 11 & $5(45 \%)$ \\
Subserosa and serosa & 15 & $11(73 \%)$ \\
\hline
\end{tabular}

\section{Discussion}

The pathogenesis of primary and secondary high grade gastric lymphomas, and the differences between them, are interesting issues. Some investigators have categorised gastric DLL among the MALT lymphomas, ${ }^{14-16}$ but others have regarded them as being separate from high grade MALT lymphomas. ${ }^{17}$ Chan et al have recently reported that DLL are associated with abnormalities of chromosome 12 , but these changes were found less often in high grade MALT lymphomas. ${ }^{18}$ Ott et al also recently pointed out that gains of chromosome 7 and 12 tend to be associated with DLL, and gains of chromosome 18 are more closely related to high grade MALT lymphomas, though a statistical difference could not be shown. ${ }^{19}$ These findings may indicate that DLL and high grade MALT lymphomas have a different pathogenesis.

We focused on the clinicopathological features and differences between low and high grade MALT lymphomas and DLL. Our data indicate that low grade MALT lymphomas have a female preponderance, like MALT lymphomas at other sites ${ }^{11}$ but DLL appears to have a slight male preponderance (table 1), though the differences did not achieve statistical significance. The regional distribution of low grade and high grade MALT lymphomas was much wider than that of DLL (figs 4 and 5). The stump invasion of lymphoma cells was detected only in MALT lymphomas. This finding is probably correlated with the wider distribution of MALT lymphomas. Lymph node metastasis was obviously dependent on the depth of the lesions, and there was a clear borderline between the submucosa and the muscle layers proper (table 2). These findings should be useful for image analyses of gastric lymphoma cases.

The mean age of patients with high grade MALT lymphoma was 8.6 years older than that of patients with low grade MALT lymphoma, and this difference was statistically significant (table 1, fig 2). There is at present no information about the time course of malignant transformation from low MALT lymphoma into high MALT lymphoma-that is, secondary high grade MALT lymphoma. Though this information could be obtained from prospective studies, the opportunities for such studies are limited. As our cases were consecutive, the age differences found may well be related to the rate of progression, which is another important result.

As shown in fig 2, the age distribution curve of low grade MALT lymphoma is normally distributed, with a peak age in the sixties. In the high grade MALT lymphoma group, the number of cases gradually increased from the fifties to the seventies. The age distribution curve of the DLL group was quite different, being much more widely distributed over the age range, and the shape of the curve suggested that the patients formed a mixed population, with a normally distributed older age group and a separate, smaller group of younger patients. Interestingly, there appeared to be a male predominance in patients younger than 50 years old. The differences in the clinicopathological features in our series suggest that some at least of the DLL cases do not originate from transformation of low grade MALT lymphomas, especially in the younger age group. This information may prove useful in the clinical management of gastric lymphomas.

We thank Ms Yoshiko Sakamoto, Mr Ryouichi Domatsu, Ms Hiromi Nakamura, and Ms Mie Kosaka for technical assistance, and Ms Yoshiko Narasaki for secretarial work. This work was supported, in part, by a grant-in-aid for scientific research from supported, in part, by a grant-in-aid for scientific research from
the Ministry of Education, Science, Sports, and Culture of the Ministry of Education, Science, Sports, and Culture of
Japan. Presented in part at the 6 th International Conference on Japan. Presented in part at the 6th International Conference on
Malignant Lymphoma at Lugano, Switzerland, June 5-8, 1997, and at the Non-Hodkin's Lymphoma Classification Project meeting at Omaha, September 8-10, 1997.

1 Isaacson PG, Wright D. Malignant lymphoma of mucosaassociated lymphoid tissue. Cancer 1983;52:1410-16.

2 Harris NL, Jaffe ES, Stein H, et al. A revised EuropeanAmerican classification of lymphoid neoplasms: a proposal from the international lymphoma study group. Blood 1994; 84:1361-92.

3 Wotherspoon AC, Ortiz HC, Falzon MR, et al. Helicobacter pylori-associated gastritis and primary B-cell gastric pylori-associated gastritis and prim

4 Wotherspoon AC, Doglioni C, Diss TC, et al. Regression of primary low-grade B-cell gastric lymphoma of mucosaassociated lymphoid tissue type after eradication of Helicobacter pylori. Lancet 1993;342:575-7.

5 Roggero E, Zucca E, Pinotti G, et al. Eradication of Helicobacter pylori infection in primary low-grade gastric lymphoma of mucosa-associated lymphoid tissue. Ann Intern Med 1995;122:767-9.

6 Bayerdorffer E, Neubauer A, Rudolph B, et al. Regression of primary gastric lymphoma of mucosa-associated lymphoid issue type after cure of Helicobacter pylori infection. MALT Lymphoma Study Group. Lancet 1995;345:1591-4.

7 Isaacson PG. Recent developments in our understanding of gastric lymphomas. Am f Surg Pathol 1996;20(suppl 1):S1-7

8 Montalban C, Manzanal A, Castrillo JM, et al. Low grade gastric B-cell MALT lymphoma progressing into high grade lymphoma. Clonal identity of the two stages of the tumour, unusual bone involvement and leukemic dissemi-

Du M, Peng H, Singh N, et al. The accumulation of p53 abnormalities is associated with progression of mucosaabnormalities is associated with progression of mucosa-
associated lymphoid tissue lymphoma. Blood 1995;86: associated

10 Omonishi K, Yoshino T, Sakuma I, et al. BCL-6 protein is identified in high-grade but not low-grade mucosaassociated lymphoid tissue (MALT) lymphomas of the stomach. Mod Pathol 1998;11:181-5.

11 Isaacson P, Norton A. Extranodal lymphomas. New York: Churchill Livingstone, 1994.

12 Ortiz HC, Wright DH. The morphological spectrum of monocytoid B-cell lymphoma and its relationship to monocytoid B-cell lymphoma and its relationship to pathology 1992;21:555-61.

13 Isaacson PG, Wotherspoon AC, Diss T, et al. Follicular colonization in B-cell lymphoma of mucosa-associated lymphoid tissue. Am f Surg Pathol 1991;15:819-28.

14 Taal G, Boot H, van Heerde P, et al. Primary non-Hodgkin's lymphomas of the stomach: endoscopic pattern and prognosis in low versus high grade malignancy in relation to the MALT concept. Gut 1996;39:556-61.

15 Krol AD, Hermans J, Kramer MH, et al. Gastric lymphomas compared with lymph node lymphomas in a populationbased registry differ in stage distribution and dissemination pattern but not in patient survival. Cancer 1997;79:390-7.

16 Nakamura S, Yao T, Aoyagi K, et al. Helicobacter pylori and primary gastric lymphoma. A histopathologic and immunohistochemical analysis of 237 patients. Cancer 1997;79:3-11.

17 Cogliatti SB, Schmid U, Schumacher U, et al. Primary B-cell gastric lymphoma: a clinicopathological study of 145 patients. Gastroenterology 1991;101:1159-70.

18 Chan W-Y, Wong N, Chan AB-W, et al. Consistent copy number gain in chromosome 12 in primary diffuse large cell lymphomas of the stomach. Am f Pathol 1998;152:11-16.

19 Ott G, Kalla J, Steinhoff A, et al. Trisomy 3 is not a common feature in malignant lymphomas of mucosa-associated lymphoid tissue type. Am F Pathol 1998;153:689-94. 